LA W RENCE LIVERMORE N A TIO NAL LABORATORY
Estimates and

Recommendations for Coincidence Geometry

W. Younes, J. J. Ressler

January 16, 2014 
This document was prepared as an account of work sponsored by an agency of the United States government. Neither the United States government nor Lawrence Livermore National Security, LLC, nor any of their employees makes any warranty, expressed or implied, or assumes any legal liability or responsibility for the accuracy, completeness, or usefulness of any information, apparatus, product, or process disclosed, or represents that its use would not infringe privately owned rights. Reference herein to any specific commercial product, process, or service by trade name, trademark, manufacturer, or otherwise does not necessarily constitute or imply its endorsement, recommendation, or favoring by the United States government or Lawrence Livermore National Security, LLC. The views and opinions of authors expressed herein do not necessarily state or reflect those of the United States government or Lawrence Livermore National Security, LLC, and shall not be used for advertising or product endorsement purposes.

This work performed under the auspices of the U.S. Department of Energy by Lawrence Livermore National Laboratory under Contract DE-AC52-07NA27344. 


\title{
Estimates and recommendations for coincidence geometry
}

\author{
W. Younes and J. J. Ressler
}

(Dated: May 23, 2013)

\section{ROUGH ESTIMATE OF COINCIDENCE SUMMING CORRECTIONS}

\section{A. Introduction}

When two truly coincident gamma-rays deposit their energy within the same detector, a composite pulse which is indistinguishable from one due to a single event may be recorded by that detector. This summing effect is known to become more important as the distance from source to detector is decreased [1]. In this short report, we give a rough estimate for the size of this effect as a function of source-to-detector distance. The formalism used in this report is taken mainly from [2], and similar results can also be found, e.g., in [1,3,4]. In general, the size of the effect will depend on the exact level scheme of the nucleus studied, but for the sake of extracting numerical values, we will assume a particular level scheme in this report.

\section{B. Assumed measurement conditions}

\section{Simplified level scheme}

For demonstration purposes, we assume the simple level scheme shown in Fig. 3 of [2], and reproduced as Fig. 1 of this report for easy reference. The energy of each gamma-ray is denoted by $E_{i}$, and its emission probability is given by $p_{i}$. The side-feeding probabilities are $\beta_{i}$, with $\beta_{1}+\beta_{2}+\beta_{3}=1$. Thus, if we denote by $b_{i}$ the fraction of decays from the level from which $\gamma_{i}$ originates and which produces the $\gamma_{i}$ photons (i.e., the branching ratios), we have the following relations[5]

$$
\begin{aligned}
& p_{1}=\beta_{2} b_{1} \\
& p_{2}=\left(\beta_{2} b_{1}+\beta_{1}\right) b_{2} \\
& p_{3}=\beta_{2} b_{3}
\end{aligned}
$$

In the numerical applications below, we will assume $\beta_{1}=0$, and $b_{1}=2 / 3, b_{2}=1, b_{3}=1 / 3$, and therefore

$$
\begin{aligned}
p_{1} & =\frac{2}{3} \beta_{2} \\
p_{2} & =\frac{2}{3} \beta_{2} \\
p_{3} & =\frac{1}{3} \beta_{2}
\end{aligned}
$$

We do not need to supply a numerical value for $\beta_{2}$, because the emission probabilities $p_{i}$ will always appear as ratios in the calculations below.

\section{Simplified detector}

In order to simplify the efficiency calculations, we approximate the Ge crystal by a flat disk (rather than a thick cylinder) of diameter $a$. For numerical applications, we will use $a=11.4 \mathrm{~cm}$ (the diameter for the BE6530 model). A schematic representation of this detector, located at a distance $d$ from a source, is shown in Fig. 2. The solid angle subtended by the disk is given by

$$
\Omega=\iint \frac{\hat{n} \cdot d \vec{a}}{r^{2}}
$$

where $\hat{n}$ is a unit vector from the origin to a point on the surface, $d \vec{a}$ is a vector whose magnitude is a differential element of area and whose direction is perpendicular to the surface. Finally, $r$ is the distance from the origin to a point 


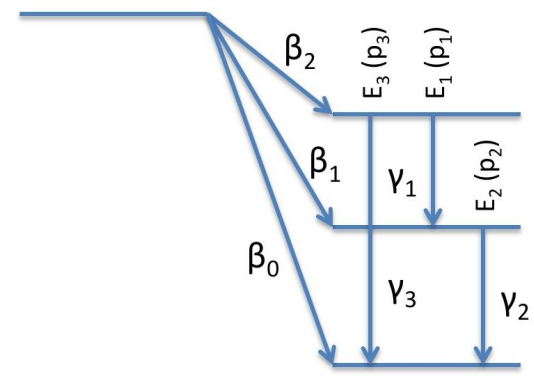

Figure 1: Simplified level scheme used in this report.

on the surface. In the special case of the disk in Fig. 2 this reduces to the double integral in cylindrical coordinates

$$
\Omega=\int_{0}^{2 \pi} d \theta \int_{0}^{a / 2} \rho d \rho \frac{\cos \phi}{d^{2}+\rho^{2}}
$$

which can be evaluated analytically

$$
\begin{aligned}
\Omega & =2 \pi d \int_{0}^{a / 2} \frac{\rho d \rho}{\left(d^{2}+\rho^{2}\right)^{3 / 2}} \\
& =2 \pi\left(1-\frac{1}{\sqrt{1+b^{2}}}\right)
\end{aligned}
$$

and where we have introduced the dimensionless parameter

$$
b \equiv \frac{a}{2 d}
$$

Given a diameter $a$ and distance $d$ of the detector, we can therefore readily calculate its geometric efficiency

$$
\varepsilon_{\text {geo }} \equiv \frac{\Omega}{4 \pi}=\frac{1}{2}\left(1-\frac{1}{\sqrt{1+b^{2}}}\right)
$$

The intrinsic efficiency $\varepsilon_{\text {int }}$ will normally depend on the gamma-ray energies, but since these will vary depending on the nucleus under consideration, we will assume that it is independent of gamma-ray energy, and in numerical applications below we will take $\varepsilon_{\text {int }}=0.6$. We will also make a distinction between the peak efficiency $\left(\varepsilon_{i}\right.$ with $i=1,2,3)$ for detecting a gamma-ray at a precise energy, and the total efficiency $\left(\varepsilon_{i}^{T}\right.$ with $\left.i=1,2,3\right)$ for detecting a gamma-ray at any energy in the spectrum (e.g., if it does not deposit its full energy in the spectrometer). In this case, we have the relations

$$
\varepsilon_{i}=\varepsilon_{\text {geo }} \times \varepsilon_{\text {int }}
$$

and

$$
\varepsilon_{i}^{T}=\frac{\varepsilon_{i}}{P / T}
$$

where $P / T$ is the peak-to-total ratio which, for numerical applications, we will take equal to 0.7 .

\section{Calculation of correction and uncertainty \\ 1. Formulas for coincidence-sum correction factors}

We now recapitulate the results in [2]. For $\gamma_{1}$, if there were no cascade, and given an activity $A$ of the nucleus feeding the levels in Fig. 1, we would expect a count rate

$$
N_{1}=A p_{1} \varepsilon_{1}
$$




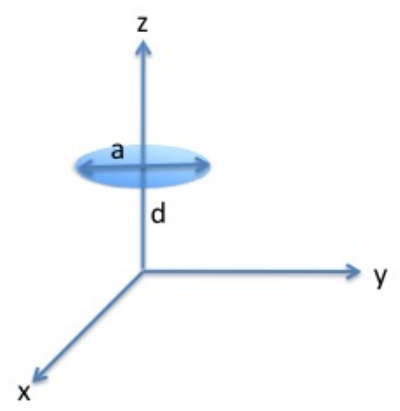

Figure 2: Disk-shaped detector of diameter $a$, at a distance $d$ from the source.

for the photopeak of this gamma-ray. However, every time $\gamma_{1}$ is emitted, $\gamma_{2}$ will also be emitted. Thus, there is a probability that the energy of $\gamma_{2}$ will also be recorded (in part or in full) at the same time as that of $\gamma_{1}$. For simplicity, we ignore any angular correlation effects. Then, the number of times we record simultaneously the sum of $E_{1}$ and either all or part of $E_{2}$ is given by $N_{1} \varepsilon_{2}^{T}$. Whenever this happens, we lose counts in the $\gamma_{1}$ photopeak, and observe a smaller number of counts

$$
N_{1}^{\prime}=N_{1}-N_{1} \varepsilon_{2}^{T}
$$

This loss must be accounted for by a correction factor

$$
C_{1} \equiv \frac{N_{1}}{N_{1}^{\prime}}=\frac{1}{1-\varepsilon_{2}^{T}}
$$

Next, for $\gamma_{2}$, if there were no cascade, we would expect a count rate

$$
N_{2}=A p_{2} \varepsilon_{2}
$$

Because of the $\gamma_{1}-\gamma_{2}$ coincidence, we expect events were the sum of $E_{2}$ and either all or part of $E_{1}$ is recorded, taking counts away from the $\gamma_{2}$ photopeak. Again, ignoring angular-correlation effects, these sum-coincidence events will occur at a rate $\left(A p_{1} \varepsilon_{1}^{T}\right) \varepsilon_{2}$, and therefore we will observe a rate in the $\gamma_{2}$ photopeak of

$$
\begin{aligned}
N_{2}^{\prime} & =N_{2}-\left(A p_{1} \varepsilon_{1}^{T}\right) \varepsilon_{2} \\
& =N_{2}\left(1-\frac{p_{1}}{p_{2}} \varepsilon_{1}^{T}\right)
\end{aligned}
$$

The loss in photopeak counts must be accounted for by the correction factor

$$
C_{2} \equiv \frac{1}{1-\frac{p_{1}}{p_{2}} \varepsilon_{1}^{T}}
$$

Finally, for $\gamma_{3}$, the situation is slightly different. In this case, it is possible to detect the full-energy sum $E_{1}+E_{2}$ from a $\gamma_{1}-\gamma_{2}$ coincidence which would be erroneously attributed to a $\gamma_{3}$ decay, thereby leading to a peak gain in the 
$\gamma_{3}$ photopeak. The observed counts, taking this effect into account, would be[6]

$$
\begin{aligned}
N_{3}^{\prime} & =N_{3}+A p_{1} \varepsilon_{1} \varepsilon_{2} \\
& =N_{3}\left(1+\frac{p_{1} \varepsilon_{1} \varepsilon_{2}}{p_{3} \varepsilon_{3}}\right)
\end{aligned}
$$

giving a peak-gain correction factor

$$
C_{3} \equiv \frac{1}{1+\frac{p_{1} \varepsilon_{1} \varepsilon_{2}}{p_{3} \varepsilon_{3}}}
$$

\section{Uncertainties for coincidence-sum correction factors}

As stated in section IB 2, we will make the simplifying assumption that the peak and total efficiencies are independent of gamma-ray energy, i.e., $\varepsilon_{1}=\varepsilon_{2}=\varepsilon_{3} \equiv \varepsilon$ and $\varepsilon_{1}^{T}=\varepsilon_{2}^{T}=\varepsilon_{3}^{T} \equiv \varepsilon^{T}$. In that case, the correction factors derived in section $\mathrm{IC} 1$ reduce to

$$
\begin{aligned}
C_{1} & =\frac{1}{1-\varepsilon^{T}} \\
C_{2} & =\frac{1}{1-\frac{p_{1}}{p_{2}} \varepsilon^{T}} \\
C_{3} & =\frac{1}{1+\frac{p_{1}}{p_{3}} \varepsilon}
\end{aligned}
$$

The variance of $C_{1}$ is given by

$$
\sigma_{C_{1}}^{2}=\left(\frac{\partial C_{1}}{\partial \varepsilon^{T}}\right)^{2} \sigma_{\varepsilon^{T}}^{2}
$$

and, after straightforward calculations we get

$$
\frac{\sigma_{C_{1}}}{C_{1}}=\underbrace{\left(\frac{\varepsilon^{T}}{1-\varepsilon^{T}}\right)}_{\rho_{1}} \frac{\sigma_{\varepsilon^{T}}}{\varepsilon^{T}}
$$

This very convenient form relates the relative uncertainty in $C_{1}$ to the relative uncertainty in the total efficiency $\varepsilon^{T}$ through a scale factor $\rho_{1}$. We can obtain similar expressions for the other correction factors (making the reasonable assumption that efficiencies and branching ratios are uncorrelated). To wit,

$$
\frac{\sigma_{C_{2}}}{C_{2}}=\underbrace{\left(\frac{\frac{p_{1}}{p_{2}} \varepsilon^{T}}{1-\frac{p_{1}}{p_{2}} \varepsilon^{T}}\right)}_{\rho_{2}} \sqrt{\left(\frac{\sigma_{p_{1}}}{p_{1}}\right)^{2}+\left(\frac{\sigma_{p_{2}}}{p_{2}}\right)^{2}+\left(\frac{\sigma_{\varepsilon^{T}}}{\varepsilon^{T}}\right)^{2}}
$$

and

$$
\frac{\sigma_{C_{3}}}{C_{3}}=\underbrace{\left(\frac{\frac{p_{1}}{p_{3}} \varepsilon}{1+\frac{p_{1}}{p_{3}} \varepsilon}\right)}_{\rho_{3}} \sqrt{\left(\frac{\sigma_{p_{1}}}{p_{1}}\right)^{2}+\left(\frac{\sigma_{p_{2}}}{p_{2}}\right)^{2}+\left(\frac{\sigma_{\varepsilon}}{\varepsilon}\right)^{2}}
$$

Ideally, the experiment should be designed so that $\rho_{i} \ll 1$, and that therefore the coincidence-sum corrections contribute very little to the overall uncertainty, compared to the efficiencies and branching ratios. 


\begin{tabular}{|c|c|}
\hline Parameter & Value \\
\hline \hline$a$ & $11.4 \mathrm{~cm}$ \\
\hline$p_{1}$ & $\frac{2}{3} \beta_{2}$ \\
\hline$p_{2}$ & $\frac{2}{3} \beta_{2}$ \\
\hline$p_{3}$ & $\frac{1}{3} \beta_{2}$ \\
\hline$\varepsilon_{\text {int }}$ & 0.6 \\
\hline$P / T$ & 0.7 \\
\hline
\end{tabular}

Table I: Parameter values used in the numerical calculations.

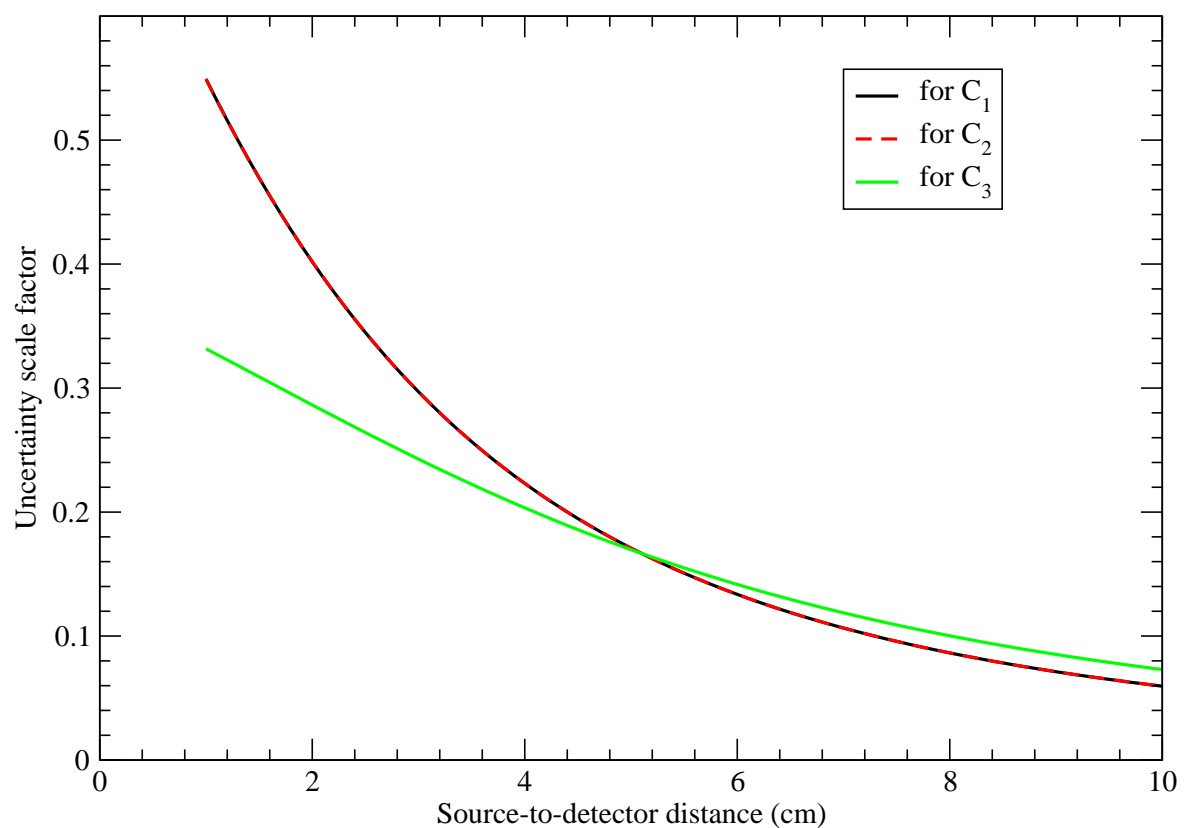

Figure 3: Uncertainty scale factors $\rho_{i}$ plotted as a function of the distance $d$ between source and detector. Note that, because $p_{1}=p_{2}$ and all the efficiencies are the same in our example, the curves for $C_{1}$ and $C_{2}$ lie on top of each other.

\section{Numerical application}

Here we plot the uncertainty scale factors $\rho_{i}$ derived in section IC 2, using the particular values of the parameters given in sections IB 1 and IB 2, and summarized in table I. These uncertainty scale factors are plotted in Fig. 3.

\section{Recommendations}

The results obtained in this report and summarized in Fig. 3 depend sensitively on the particular level scheme under consideration. We note in particular, that the factor $\rho_{3}$ tends to its maximum value of 1 for $b_{1} \gg b_{3}$. In other words, if the $\gamma_{3}$ branch in Fig. 1 is very weak, then whenever we record an energy $E_{3}$ in the detector, it is far more likely to be caused by the $\gamma_{1}-\gamma_{2}$ summed coincidence, with energy $E_{1}+E_{2}=E_{3}$, than by an actual $\gamma_{3}$ photon. In that case, the uncertainty contribution from the peak-gain correction factor $C_{3}$ would be as large as that of the other sources of uncertainty in the problem. In our particular example, we find that the correction uncertainties become small (i.e., $\rho_{i} \sim 10 \%$ ) for a distance $d \gtrsim 10 \mathrm{~cm}$. In practice, the distance $d$ should be selected with the specific gamma-rays of interest and associated decay schemes in mind, with the goal of maximizing the geometric efficiency while keeping the coincidence-sum corrections at an acceptably small level. 


\section{SCATTER}

\section{A. Introduction}

The detector will be sensitive the gamma-rays emitted by the source, as well as any photons scattered into the detector volume from surrounding materials. The more material surrounding the detection system, the large the scatter contribution to the energy spectrum. The energy of the scattered photon $\left(E^{\prime}\right)$ depends on the energy of the originating gamma-ray $(E)$ as well as the scattering angle $(\theta)$ :

$$
E^{\prime}=\frac{E}{1+\frac{(1-\cos \theta) E}{m_{e} c^{2}}}
$$

where $m_{e}$ is the rest mass of the electron $(0.511 \mathrm{MeV})$. Higher angle scatter, $>90^{\circ}$, can significantly increase the count contribution to the lower energies of the gamma-ray spectrum. Very high scatter, at $\sim 180^{\circ}$, produces a broad backscatter peak that may confuse peak fitting, activity estimates, and nuclide identification efforts.

\section{B. Recommendation}

With active samples, significant scatter from shielding materials near the detector volume can hinder spectroscopic analyses. Therefore, the detectors should not have any additional materials nearby.

[1] G. J. McCallum and G. E. Coote, Nucl. Instr. Meth. 130, 189-197 (1975).

[2] K. Debertin and U. Schötzig, Nucl. Instr. Meth. 158, 471-477 (1979).

[3] R. J. Gehrke, R. G. Helmer, and R. C. Greenwood, Nucl. Instr. Meth. 147, 405-423 (1977).

[4] S. I. Vasil'ev, et al., Instr. Expt. Tech. 49, 34-40 (2005).

[5] We ignore conversion electrons in the present estimates, but a correction for this effect could also be included in the formalism (see, e.g., [1]).

[6] Note that we use the peak efficiencies $\varepsilon_{i}$ instead of the total efficiencies $\varepsilon_{i}^{T}$ here because we are only interested in gamma-rays that deposit their full energy. 